
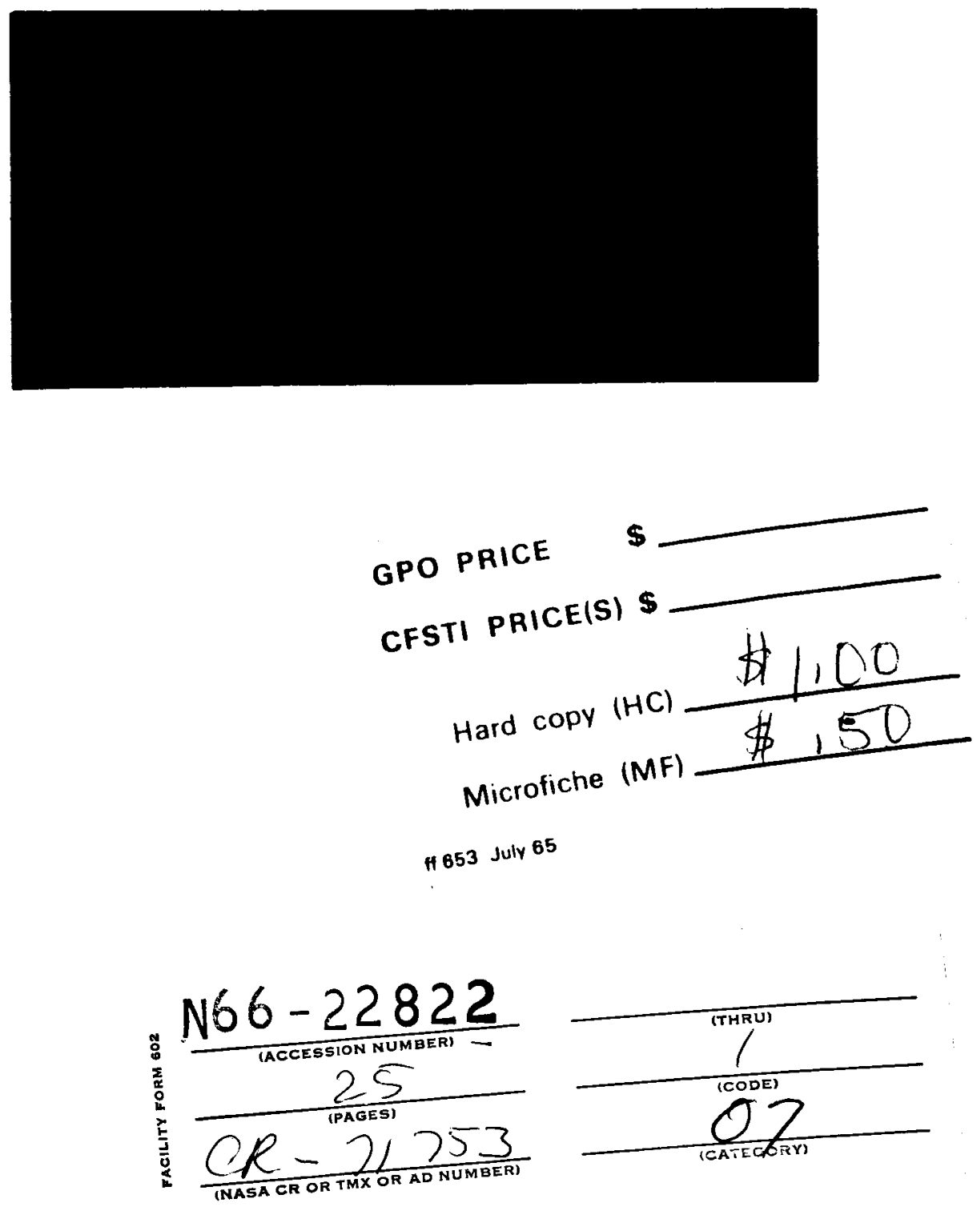

CRES \|\|\|\|$\| \begin{aligned} & \text { THE UNIVERSITY OF KANSAS • CENTER FOR RESEARCH INC } \\ & \text { ENGINEERING SCIENCE DIVISION • LAWRENCE, KANSAS }\end{aligned}$ 
Technical Report

$61-2$

Plane Wave Scattering from a

Rough Surface with Correlated Large and

Small Scale Orders of Roughness

\author{
May, 1965 \\ H. S. Hayre \\ and \\ D. E. Kaufman \\ Department of Electrical Engineering \\ Kansas State University \\ Manhattan, Kansas
}





\section{ABSTRACT}

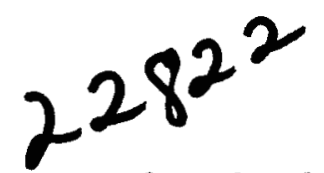

A rough surface is assumed to be composed of a slowly varying large-scale roughness, and a rapidly varying smallscale roughness, and that these are correlated. The exponential form of both the auto-correlations of these surfaces and their cross-correlation were used. This paper shows that the return from such a composite surface for a plane wave incidence can have either one of the following terms contribute a major portion of the total return power, namely the large scale roughness term, small scale roughness term and the cross-correlation term. It is therefore concluded that contribution due to each term must be evaluated before neglecting its effect on the total return power. These results are equally applicable to acoustic and radar cases. 


\section{Introduction}

A number of rough surface models have been proposed in the analysis of electromagnetic wave scattering from a rough surface. The simplest model is that of a surface, $\zeta(x, y)$ where roughness, i.e., deviation from the mean surface height, can be expressed by a single statistical distribution. A better model of the rough surfaces often met in practical applications is to consider these surfaces as the superposition of several types of roughness. For example, terrain may consist of such large scale features as rocks and mountains on which are superposed small scale roughnesses due to rock, vegetation, etc. As another example, the typical sea surface is comprised of "swell", "sea", and "ripple". For the case of radar return from the moon and planets several papers $(1,2)$ have suggested that small scale roughness, rather than large scale, may play an important part in the backscatter signal.

Beckmann (3) has considered such a composite rough surface model where the surface $\zeta(x, y)$ is the superposition of $n$ types of roughnosses

$$
\zeta(x, y)=\zeta_{1}(x, y)=\zeta_{2}(x, y)+\ldots+\zeta_{n}(x, y)
$$

where $\zeta_{1}, \zeta_{2} \ldots \zeta_{\mathrm{n}}$ are independent stationary random functions each with its own statistical distribution and correlation function. However, natural evidence about the surface of the earth, and perhaps the surfaces of the planets, indicates that small scale roughnesses as caused by such factors as wind or water erosion, landslides or rockfalls, and artificial handling 
are definitely dependent upon the local large scale roughness.

In' light of such natural evidence this paper will investigate scattering from a composite rough surface where the large and the small scale roughness are correlated. Although this paper deals with a surface composed of two dependent random processes, the procedures used can be readily extended to any number of dependent random processes that might constitute the surface.

\section{General Theory}

The general Kirchoff solution for a perfectly conductive rough surface whose mean value coincides with the $x-y$ plane and whose radii of curvature are always greater than the incident wavelength is, according to Beckmann (4):

$$
E\left(\theta_{1} ; \theta_{2} ; \theta_{3}\right)=\frac{E_{N} F}{A} \int_{-X}^{Y} \int_{-Y}^{Y} \exp [i \vec{i} \cdot \vec{r}] d x d y
$$

The scattering geometry is as shown in Figure 1. Furthermore, $A=4 X Y$, the area of the scattering surface; $\vec{k}_{1}$ and $\vec{k}_{2}$ are the propagation vectors of the incident and scattered waves. $\overrightarrow{\mathrm{v}}=\overrightarrow{\mathrm{k}}_{1}-\overrightarrow{\mathrm{k}}_{2}$, with rectangular components:

$$
\begin{aligned}
v_{x} & =\frac{2 \pi}{\lambda}\left(\sin \theta_{1}-\sin \theta_{2} \cos \theta_{3}\right) \\
v_{y} & =\frac{-2 \pi}{\lambda}\left(\sin \theta_{2} \sin \theta_{3}\right) \\
v_{z} & =\frac{-2 \pi}{\lambda}\left(\cos \theta_{1}+\cos \theta_{2}\right) \\
\vec{r} & =\overrightarrow{x i}+\overrightarrow{y j}+(x, y) \vec{k}, \text { the radius vector to any point }
\end{aligned}
$$

in the rough surface. 


$$
F=\frac{1+\cos \theta_{1} \cos \theta_{2}-\sin \theta_{1} \sin \theta_{2} \cos \theta_{3}}{\cos \theta_{1}+\cos \theta_{2}}
$$

$E_{N}=\frac{i k e^{i k R_{O A}}}{2 \pi R_{O}}$, the amplitude of the field reflected by a smooth plane surface of area $A$ at distance $R_{0}$ for normai incidence of a unit plane wave, $E_{1}=e^{i\left(\vec{k}_{1} \cdot \vec{r}\right)}$.

In the derivation of (1) edge effect terms are neglected for it is assumed $A \gg>\lambda^{2}$; depolarization effects are ignored by considering only the scalar value of $\vec{E}_{2}$ as the question of direction of $\vec{E}_{2}$ is more difficult to solve. Experimental measurements indicate that the scattered field is largely determined by surface roughness rather than by surface conductivity, so the assumption of perfect conductivity for the rough surface is not unduly restrictive.

The surface roughness $\zeta(x, y)$ will be considered as the sum of a large scale roughness $\zeta_{L}$ and a small scale roughness $\zeta_{S}$ (see Figure 2); $\zeta(x, y)=\zeta_{L}(x, y)+\zeta_{S}(x, y)$

Each roughness component is assumed to have zero mean value.

Now the r.m.s. scattered power, $\left\langle E_{2} E_{2} *\right\rangle$ is given by

$$
\begin{aligned}
& \left\langle E_{2} E_{2} *\right\rangle=\frac{E_{N}^{2} F^{2}}{A} \int_{-X}^{X} \int_{-X}^{X} \int_{-Y}^{Y} \int_{-Y}^{Y} \exp \left[i v_{x}\left(x_{1}-x_{2}\right)+i v_{Y}\left(y_{1}-y_{2}\right)\right] \\
& \left\langle e^{i v}\left(\zeta_{1}-\zeta_{2}\right)\right\rangle_{d x_{1} d x_{2} d y_{1} d y_{2}}
\end{aligned}
$$

$$
\text { where } \zeta_{1}=\zeta\left(x_{1}, y_{1}\right) \quad \zeta_{2}=\zeta\left(x_{2}, y_{2}\right)
$$


By introducing a separation parameter, $\tau$, defined as

$$
\tau=\left[\left(\mathrm{x}_{2}-\mathrm{x}_{1}\right)^{2}+\left(\mathrm{y}_{2}-\mathrm{y}_{1}\right)^{2}\right]^{1 / 2}
$$

equation (3) becomes:

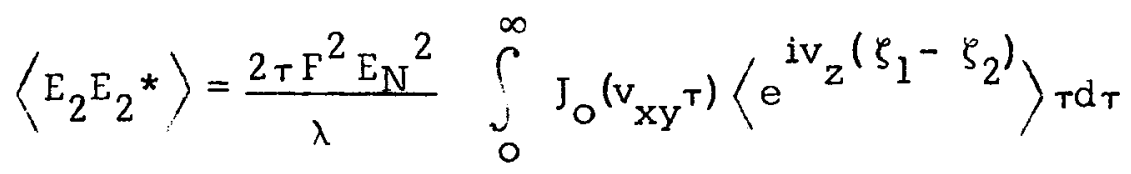

where $J_{0}\left(v_{x y} \tau\right)$ is the Bessel function of the first kind and order zero with argument $v_{x y} T=\tau\left(v_{x}^{2}+v_{y}{ }^{2}\right)^{1 / 2}$. Since only the regions near $\tau=0$ contribute to the integral, the range of integration in (5) has, for convenience, been made infinite.

Now $\left\langle e^{i v_{z}\left(\zeta_{1}-\zeta_{2}\right)}\right\rangle=\left\langle e^{i v_{z}\left[\left(z_{1}+z_{3}\right)-\left(z_{2}+z_{4}\right)\right.}\right\rangle=$

$\int_{-\infty}^{\infty} \int_{-\infty}^{\infty} \int_{-\infty}^{\infty} \int_{-\infty}^{\infty} w_{4}\left(z_{1}, z_{2}, z_{3}, z_{4}\right) e^{i v_{z}\left[\left(z_{1}+z_{3}\right)-\left(z_{2}+z_{4}\right)\right]} d z_{1} d z_{2} d z_{3} d z_{4}$

with

$$
\begin{array}{ll}
z_{1}=\zeta_{S}\left(x_{1}, y_{1}\right) & z_{2}=\zeta_{S}\left(x_{2}, y_{2}\right) \\
z_{3}=\zeta_{L}\left(x_{1}, y_{1}\right) & z_{4}=\zeta_{L}\left(x_{2}, y_{2}\right)
\end{array}
$$

$W_{4}\left(z_{1}, z_{2}, z_{3}, z_{4}\right)$ is the four dimensional probability density function for the variables $\mathrm{Z}_{1} \cdot \mathrm{Z}_{2} \cdot \mathrm{Z}_{3} \cdot \mathrm{z}_{4} \cdot$

If $\mathrm{Z}_{1}, \mathrm{Z}_{2}, \mathrm{Z}_{3}$, and $\mathrm{Z}_{4}$ are normally distributed and statistically dependent random variables, $W_{4}$ takes the form:

$W_{4}\left(Z_{1}, Z_{2}, Z_{3}, Z_{4}\right)=(2 \pi)^{-2}|M|^{\frac{1}{2}} \exp \left(-\frac{1}{2|M|} \sum_{i=1}^{4} \sum_{j=1}^{4} M_{i j} Z_{i} Z_{j}\right)$

In the above expression $|M|$ is the determinant of the matrix $[M]$ that has as its $i-j{ }^{\text {th }}$ element, $d_{i j}=\left\langle z_{i} z_{j}\right\rangle$, the second moment 
of the random variables $Z_{i}$ and $Z_{j}$, and $M_{i j}$ is the cofactor of $\mathrm{d}_{\mathrm{ij}}$ in the determinant $|\mathrm{M}|$.

Evaluation of the $d_{i j}$ gives

$$
[M]=\left[\begin{array}{llll}
\sigma_{S}{ }^{2} & \sigma_{S}{ }^{2} \rho_{S}(\tau) & \sigma_{S} \sigma_{L} \rho_{S L}(0) & \sigma_{S} \sigma_{L} \rho_{S L}(\tau) \\
\sigma_{S}{ }^{2} \rho_{S}(\tau) & \sigma_{S}{ }^{2} & \sigma_{S} \sigma_{L} \rho_{S L}(\tau) & \sigma_{S} \sigma_{L} \rho_{S L}(0) \\
\sigma_{S} \sigma_{L} \rho_{S L}(0) & \sigma_{S} \sigma_{L} \rho_{S L}(\tau) & \sigma_{L}^{2} & \sigma_{L}^{2}{ }^{2} \rho_{L}(\tau) \\
\sigma_{S} \sigma_{L} \rho_{S L}(\tau) & \sigma_{S} \sigma_{L} \rho_{S L}(0) & \sigma_{L}{ }^{2} \rho_{L}(\tau) & \sigma_{L}{ }^{2}
\end{array}\right]
$$

where $\sigma_{\mathrm{S}}$ and $\sigma_{\mathrm{L}}$ are the standard deviations of $\zeta_{\mathrm{S}}$ and $\zeta_{\mathrm{L}}$ ' $\rho_{S}(\tau)$ and $\rho_{L}(\tau)$ are the normalized auto-correlation functions of $\zeta_{S}$ and $\zeta_{L}$, and $\rho_{S L}(\tau)$ is the normalized cross-correlation function of $\zeta_{S}$ and $\zeta_{L}$. In the above evaluation it has been assumed that the surface is statistically isotropic, i.e., that $\left\langle\zeta_{S}\left(x_{1}, y_{1}\right) \zeta_{L}\left(x_{2}, y_{2}\right)\right\rangle=\left\langle\zeta_{L}\left(x_{1}, y_{1}\right) \zeta_{S}\left(x_{2}, y_{2}\right)\right\rangle$ Now eq. (6) may be written as:

$$
\left\langle e^{i v_{z}}\left(\zeta_{1}-\zeta_{2}\right)\right\rangle=H\left(v_{z},-v_{z}, v_{z}, v_{z}\right)
$$

where $\mathrm{H}\left(\mathrm{v}_{1}, \mathrm{v}_{2}, \mathrm{v}_{3}, \mathrm{v}_{4}\right)$ is the characteristic function of the joint probability density $\mathrm{w}_{4}\left(\mathrm{z}_{1}, \mathrm{z}_{2}, \mathrm{z}_{3}, \mathrm{z}_{4}\right)$.

For $\mathrm{W}_{4}$ of the form given by eq. (7), Middleton (5) gives the characteristic function as

$$
H\left(v_{1}, v_{2}, v_{3}, v_{4}\right)=\exp \left[-\frac{1}{2} \sum_{j=1}^{4} \sum_{k=1}^{4} v_{i} v_{k} d_{j k}\right]
$$


so that using eq. (8):

$$
\begin{aligned}
& \left\langle e^{i v_{z}\left(\zeta_{I}-\zeta_{2}\right)}\right\rangle=\left[\operatorname { e x p } \left[-v_{z}{ }^{2}\left\{\left(\sigma_{S}{ }^{2}+\sigma_{L}{ }^{2}+2 \sigma_{S} \sigma_{L} \rho_{S L}(0)\right)\right]\right.\right. \\
& \left.-\left(\sigma_{S}{ }^{2} \rho_{S}(\tau)+\sigma_{L}{ }^{2} \rho_{L}(\tau)+2 \sigma_{S} \sigma_{L} \rho_{S L}(\tau)\right\}\right]
\end{aligned}
$$

Setting,

$$
\begin{aligned}
& \sigma^{2}=\sigma_{S}{ }^{2}+\sigma_{L}{ }^{2}+2 \sigma_{S} \sigma_{L} \rho_{S L}(0) \\
& g=v_{z}{ }^{2} \sigma^{2} \\
& G(\tau)=-v_{z}{ }^{2}\left\{\sigma_{S}{ }^{2} \rho_{S}(\tau)+\sigma_{L}{ }^{2} \rho_{L}(\tau)+2 \sigma_{S} \sigma_{I} \rho_{S I}(\tau)\right\}
\end{aligned}
$$

and applying equation (11) to equation (5),

$$
\left\langle E_{2} E_{2}{ }^{*}\right\rangle=\frac{2 \pi F^{2} E_{N}}{A} \int_{0}^{\infty} J_{0}\left(V_{x y} \tau\right) \exp [G-g] T d \tau
$$

Evaluation of the Scattered Power For Slight

\section{Surface Roughness}

For slight surface roughness the following assumptions are made:

$$
\begin{aligned}
& \sigma_{\mathrm{L}} \gg \sigma_{\mathrm{S}} \\
& \frac{\sigma}{\lambda}=\frac{\sigma \mathrm{L}}{\lambda} \leq 0.035
\end{aligned}
$$

so that

$$
\begin{aligned}
& \mathrm{g}_{\text {max }}=\frac{4 \pi \sigma^{2}}{\lambda^{2}} \leq 0.2 \\
& \mathrm{G}_{\text {max }}=\mathrm{G}_{\max }(0)=\mathrm{g}_{\max } \leq 0.2
\end{aligned}
$$


In order to avoid a divergent integral it is necessary to rearrange equation (13) by noting that

$$
\left\langle E_{2} E_{2} *\right\rangle=\left\langle E_{2}\right\rangle\left\langle E_{2} *\right\rangle+D\left\{E_{2}\right\}
$$

where

$$
D\left\{E_{2}\right\}=\left\langle\left|E_{2}-\left\langle E_{2}\right\rangle\right|^{2}\right\rangle
$$

or $\mathrm{D}\left\{\mathrm{E}_{2}\right\}$ is the variance of the complex variable $\mathrm{E}_{2}$. This variance $D\left\{E_{2}\right\}$ may be written in terms of an integral over the scattering surface as

$$
D\left\{E_{2}\right\}=\frac{2 \pi F^{2} E_{N}^{2}}{A} \int_{0}^{\alpha} J_{0}\left(V_{x y} \tau\right)\left[\left\langle e^{i v_{z}\left(\zeta_{1}-\zeta_{2}\right)}\right\rangle-\left\langle e^{i v_{z} \zeta_{1}}\right\rangle\left\langle e^{i v_{z} \zeta_{2}}\right\rangle\right]_{\tau d \tau}
$$

Now,

$$
\begin{aligned}
& \left\langle e^{\left.i v_{z} \zeta_{1}\right\rangle}=\left\langle e^{i v_{z} \zeta_{2}}\right\rangle=\left\langle e^{\left.i v_{z} \zeta\right\rangle}\right\rangle\right. \\
& \left.=\int_{-\infty}^{\infty} \int_{-\infty}^{\infty}{ }^{i v} 2^{i w_{1}}, w_{2}\right) e^{i v_{z}\left(w_{1}+w_{2}\right)}{ }_{d w_{1}} d w_{2}=H\left(v_{z}, v_{z}\right)
\end{aligned}
$$

where

$$
w_{1}=\zeta_{S}(x, y), \quad w_{2}=\zeta_{L}(x, y)
$$

and

$$
H\left(v_{1}, v_{2}\right)=\exp \left(-\frac{1}{2}\left[\sigma_{S}^{2} v_{1}^{2}+2 \sigma_{S} \sigma_{L} \rho_{S L}(0)+\sigma_{L}^{2} v_{z}^{2}\right]\right)
$$


Thus be using equation (12),

$$
\left\langle e^{i v_{z} \zeta}\right\rangle=\exp \left(-\frac{1}{2} g\right)=\exp \left(-\frac{1}{2} v_{z}^{2} \sigma^{2}\right)
$$

Substituting (19) into (17), and using (13) and (16),

$$
\begin{aligned}
& D\left\{E_{2}\right\}=\frac{2 \pi F^{2} E_{N}^{2}}{A} \int_{0}^{\infty} J_{0}\left(v_{x y} \tau\right)[\exp (G-g)-\exp (-g)] \tau d \tau \\
& =\frac{2 \pi F^{2} E_{N}^{2} e^{-g} \int_{0}^{\infty} J_{0}\left(v_{x y} \tau\right)\left[\left(\sum_{m=0}^{\infty} \frac{G}{m !}-1\right)\right] \tau d \tau}{A} \sum_{m=1}^{\infty} \frac{1}{m !} \int_{0}^{\infty} J_{0}\left(v_{x y} \tau\right) G^{m} \tau d \tau
\end{aligned}
$$

The last expression was obtained by the interchange of integration and summation.

Using equation (16) the r.m.s. scattered power will be:

$$
\left\langle E_{2} E_{2}{ }^{*}\right\rangle=E_{N}^{2} e^{-g} F^{2}\left[\rho_{0}^{2}+\frac{2 \pi}{A} \sum_{m=1}^{\infty} \frac{1}{m !} \int_{0}^{\infty} J_{O}\left(v_{x y} \tau\right) G^{m} \tau d \tau\right]
$$

Since

$$
\left\langle E_{2}\right\rangle=\frac{F E_{N}}{A} \int_{-X}^{X} \int_{-Y}^{Y} e^{i}\left(v_{x} x+v_{y} z\right)\left\langle e^{i v} z^{\zeta}\right\rangle d_{X} d_{Y}=p_{O} E_{N} F e^{-\frac{g}{2}}
$$

with

$$
\rho_{0}=\frac{\sin v_{x}^{x} \sin v_{y} Y}{v_{x} v_{y} X Y}
$$


The first term, $E_{N}{ }^{2} e^{-g} F^{2} \rho_{0}^{2}$, of equation (2l) corresponds to reflection in the "specular"direction, i.e., where $\theta_{1}=\theta_{2}$, and $\theta_{3}=0$. This first term has a value only in the immediate neighborhood of the "specular" direction and is the predominant term for scattering in this direction when the surface is slightly rough.

The second or infinite series term of (21) correspona's to the diffuse scattering from the rough surface. Since the surface has slight overall roughness, $G(\tau)$ is small and only the first term of the series is required to evaluate $\mathrm{E}_{2} \mathrm{E}_{2}$ * :

$$
\left\langle E_{2} E_{2}{ }^{\prime}\right\rangle=E_{N}^{2} e^{-g} F^{2}\left[\rho_{0} 2+\frac{2 \pi}{A} \int_{0}^{\infty} J_{0}\left(v_{x y}\right) G(\tau) \tau d \tau\right]
$$

Studies of most terrain data indicate that exponential correlation functions adequately describe the large and small scale roughnesses, i.e.,

$$
\rho_{S}(\tau)=e^{-|\tau| / \beta_{S}} \quad \rho_{L}(\tau)=e^{-|\tau| / \beta_{S}}
$$

where $\beta_{S}$ and $\beta_{L}$ are the correlation distances of the large and small scale roughnesses.

The assumption will be made that the cross-correlation function is also exponential with a correlation distance of $\sqrt{\beta_{S} \beta_{L}}$,

$$
\rho_{S I}(\tau)=K e^{-|\tau| \sqrt{\beta_{S} \beta_{L}}} \quad|K| \leq 1
$$


Evaluating $G(T)$ in terms of equation (23) and (24), and applying the identity,

$$
\int_{0}^{\infty} J_{0}\left(v_{x y}\right)^{-m \tau / \beta} T d \tau=\frac{\beta^{2}}{m_{2}\left(1+v_{x y}{ }^{2} \beta^{2} / m^{2}\right)^{3 / 2}}
$$

equation (22) becomes,

$$
\begin{aligned}
& \left\langle E_{2} E_{2}{ }^{*}=E_{N}{ }^{2} e^{-g_{F}{ }^{2}}\left[\rho_{0}^{2}+\frac{2 \pi}{A} v_{z}^{2} \frac{\sigma_{S}{ }^{2} \beta_{S}^{2}}{\left(1+v_{x y}{ }^{2} \beta_{L}^{2}\right)^{3 / 2}}\right.\right. \\
& \left.+\frac{2 K \sigma_{S} \sigma_{L} \beta_{S} \beta_{L}}{\left(1+v_{x y}{ }^{2} \beta_{S} \beta_{L}\right)^{3 / 2}}+\frac{\sigma_{L}^{2} \beta_{L}^{2}}{\left(1+v_{x y}{ }^{2} \beta_{L}^{2}\right)^{3 / 2}}\right]
\end{aligned}
$$

Thus, the diffusive scattering term from a slightly rough surface has been decomposed into three terms that respectively correspond to the effect of small scale roughness alone, the effect of large scale roughnoss alone, and the interaction between large and small scale roughness.

Evaluation of the Scattered Power For Moderate Surface Roughness

For moderate surface roughness it will be assumed that

$$
\begin{aligned}
& \sigma_{\mathrm{L}} \gg \sigma_{\mathrm{S}} \\
& \frac{\sigma}{\lambda}=\frac{\sigma_{\mathrm{L}}}{\lambda} \leq 0.1
\end{aligned}
$$


so that

$$
g_{\max }=G_{\max }(\tau) \leq 1.0
$$

Using the auto-correlation and cross-correlation functions defined by $(23)$ and $(24)$, and approximating $\left\langle E_{2} E_{2} *\right.$ by the first two terms of the series, equation (21) gives

$$
\begin{aligned}
& \left\langle E_{2} E_{2}{ }^{*}=E_{N}^{2} F^{2} e^{-g}\left[\rho_{0}^{2}+\frac{2 \pi}{A} v_{z}^{2}\left(\frac{\sigma_{S}^{2} \beta_{S}^{2}}{\left(1+v_{x Y}^{2} \beta_{S}^{2}\right)^{3 / 2}}+\right.\right.\right. \\
& \left.+\frac{2 K_{\sigma_{S} \sigma_{L} \beta_{S} \beta_{L}}}{\left(1+v_{x y}{ }^{2} \beta_{S} \beta_{L}\right)^{3 / 2}}+\frac{\sigma_{L}^{2} \beta_{L}^{2}}{\left(1+v_{x y}{ }^{2} \beta_{L}^{2}\right)^{3 / 2}}\right\}+\frac{\pi}{A} v_{z}^{4}\left\{\frac{\sigma_{S}{ }^{4} \beta_{S}^{2}}{\left.4\left(1+v_{x y}{ }^{2} \beta_{S}^{2}\right)^{2}\right)^{3 / 2+}}\right. \\
& +\frac{\sigma_{L}{ }^{4} \beta_{L}{ }^{2}}{4\left(1+v_{x y}{ }^{2} \beta_{L}{ }^{2 / 4}\right)^{3 / 2}}+\frac{K_{\sigma_{S}}{ }^{2} \sigma_{L}{ }^{2} \beta_{S} \beta_{L}}{\left(1+v_{x y}{ }^{2} \beta_{L} \beta_{L / 4}\right)^{3 / 2}}+\frac{2 \sigma_{S}{ }^{2} \sigma_{L}{ }^{2} \beta_{I}{ }^{2}}{\left(1+v_{x y}{ }^{2} \beta_{1}{ }^{2}\right)^{3 / 2}} \\
& \left.\left.+\frac{4 K_{\sigma}{ }_{S}^{3} \sigma_{L}{ }^{2} \beta_{2}{ }^{2}}{\left(1+v_{x y}{ }^{2} \beta_{2}{ }^{2}\right)^{3 / 2}}+\frac{\left.4 K_{\sigma_{S}} \sigma_{L}{ }^{3} \beta_{3}{ }^{2}\right)}{\left(1+v_{x y}{ }^{2} \beta_{3}{ }^{2}\right)^{3 / 2}}\right)\right]
\end{aligned}
$$

where

$$
\hat{p}_{1}-\frac{\beta_{S}^{\beta}{ }_{L}}{\beta_{L}^{\beta} \beta_{S}} \quad \beta_{2}=\frac{\beta_{S} \sqrt{\beta_{S} \beta_{I}}}{\beta_{S}+\sqrt{\beta_{S} \beta_{L}}} \quad \beta_{3}=\frac{\beta_{I} \sqrt{\beta_{S} \beta_{I}}}{\beta_{L}+\sqrt{\beta_{S} \beta_{L}}}
$$

More terms of the series that appears in (21) could be used to calculate $\left\langle E_{2} E_{2} *\right\rangle$ for surfaces of greater roughness, but the number of terms to be evaluated soon becomes prohibitive for the series does not converge rapidly for large values of $G(\tau)$.

Evaluation of the Scattered Power for Extreme Surface Roughness

This last case finds the widest application since it best describes the roughness of most terrains. 
Here it is assumed that

$$
g_{\max }=G_{\max }(\tau)>>1 \text {. }
$$

Rather than evaluate the infinite series in (21), it is better in this case to use a saddle-point integration of (13).

Since $\sigma$ and $\sigma_{\mathrm{L}}$ are large, $\exp (\mathrm{G}-\mathrm{g})$, with $\mathrm{G}$ and $g$ defined as in (12), will have a significant value only for values of $T$ near zero. Thus, the exponential auto-correlation and cross-correlation functions of (23) and (24) are replaced by the approximations,

$$
\begin{array}{r}
\rho{ }_{S}(\tau)=1 \frac{\tau}{\beta_{S}} \quad \rho_{L}(\tau)=1-\frac{T}{L} \\
\rho_{S L}(\tau)=K\left(1-\frac{T}{\sqrt{\beta_{S} \beta_{L}}}\right)
\end{array}
$$

So that

$$
\exp (G-g)=\exp [-\tau \Sigma]
$$

with

$$
s=v_{Z}{ }^{2}\left(\frac{\sigma_{S}}{\beta \frac{\beta_{S}}{\beta_{L}}}+\frac{\sigma_{L}^{2}}{\beta_{L}}+\frac{2 \mathrm{~K} \sigma_{S} \sigma_{\mathrm{L}}}{\sqrt{\beta_{S} \beta_{L}}}\right)
$$

After completion of the integration of (13),

$$
\left\langle E_{2} E_{2} *\right\rangle=\frac{2 \pi F^{2} E_{N}^{2} \Sigma}{A\left(\Sigma^{2+2} v^{2}\right)} 3 / 2
$$

The scattering solution of (33) is essentially of the same form as given by Beckmann (3) for a composite rough surface that is the superposition of independent normal distributed random processes with exponential correlation functions. The statistical dependence of the large and small scale roughness produces just the same effect as though the rough surface consisted of three independent processes with standard deviations $\sigma_{S}, \sigma_{L}, \sqrt{2 \mathrm{~K}_{\sigma_{S}} \sigma_{L}}$, and correlation distances $\beta_{S}, \beta_{L}$, and $\sqrt{\beta_{S} \beta_{L}}$. 


\section{Conclusion}

Inspection of the results of $(25),(28)$ and (33) points out the error of neglecting small scale roughness $\frac{\sigma_{S}}{\lambda}$ and its r.m.s. slope $\frac{\sigma_{S}}{\beta_{S}}$ in any calculation of power reflected from a rough surface. Also, these results clearly show the effect of statistical dependences between the roughness components of a composite rough surface. In each of the three cases described in this paper, the correlation between large and small scale roughness produces terms in the scattered power solution that vanish when the two roughness components are independent.

The importance of the small scale roughness and the statistical dependence between large and small scale roughness is easily illustrated for an extremely rough surface. For such a terrain assume $\sigma_{S}-10 \lambda, \sigma_{L}-\sigma-1000 \lambda$ and $\sigma_{L} / \beta_{L}-0.02$. Then for various values of small scale roughness, the relative value of the three terms that comprise the factor s:appearing in equation (33) are given in the following table.

\begin{tabular}{|c|c|c|c|}
\hline \multirow{2}{*}{ RMS } & \multicolumn{3}{|c|}{ TERNIS OF $\Sigma$} \\
\cline { 2 - 4 } & $\frac{\sigma_{S}{ }^{2}}{\beta_{S}}$ & $\frac{\sigma_{L}{ }^{2}}{\beta_{L}}$ & $\frac{2 \sigma_{S} \sigma_{L}}{\sqrt{\beta_{I} \beta_{S}}}$ \\
\hline 0.1 & $1 \lambda$ & $20 \lambda$ & $9 K \lambda$ \\
1.0 & $10 \lambda$ & $20 \lambda$ & $28 \mathrm{~K} \lambda$ \\
5.0 & $50 \lambda$ & $20 \lambda$ & $64 \mathrm{~K} \lambda$ \\
\hline
\end{tabular}


Furthermore, the above results of equations (25), (23), and (33) used in previously reported work $(6,7)$ on polarized radar return and its dependence upon surface roughness parcmeters, yield useful results in the analysis of radar return from a rough surface. 


\section{REFERENCES}

1. J. V. Evans and G. V. Pettengill, "The Scattering Behavior of the Moon at Wavelength of 3.6, 68 and 784 Centimeters", J. Geophys. Res., Vol 68, No. 2, pp. 423-447, January 1963.

2. A. K. Fung and R. K. Nioore, "Theory of Radar Scatter from Rough Surfaces, Bistatic and Monostatic, with Applications to Lunar Radar Return", J. Geophys. Res. , Vol. 69, No. 6, pp. 1075-1081, March 1964 .

3. Beckmann, Peter, "Scattering by Composite Rough Surfaces", Report No. 4, University of Colorado, 1964.

4. Beckmann, Peter and Spizzichino, A., "The Scattering of Electromagnetic Waves from Rough Surfaces" Pergamon Press, Oxford, 1963, pp. 17-28.

5. Middleton, David, "An Introduction to Statistical Communication Theory", McGraw-Hill, 1960, pp. 340-343.

6. Hayre, H. S. USNC-VRSI, Fall 1963.

7. Hayre, H. S., NRL Report, (under publication). 


\section{LIST OF FIGURES}

Figure 1. Scattering Geometry, where

$$
\begin{aligned}
& \Theta_{1}=\text { Angle of Incidence } \\
& \theta_{2}, \theta_{3}=\text { Direction Scatter }
\end{aligned}
$$

Figure 2. A Rough Surface $\zeta$ Composed of Small Scale $\zeta_{L}$ and Large Scale Roughness $\zeta_{S}$. 


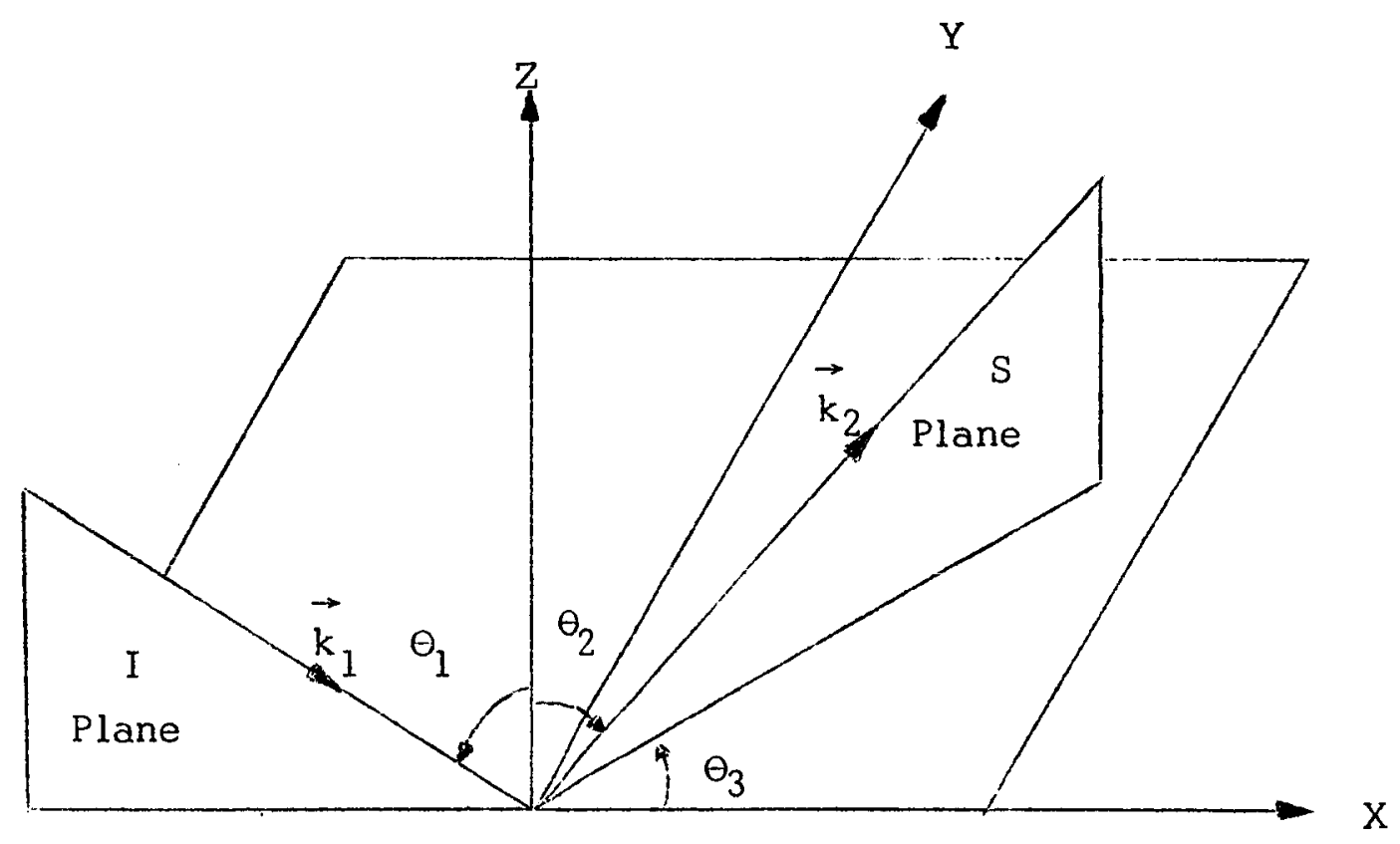

Fig. 1

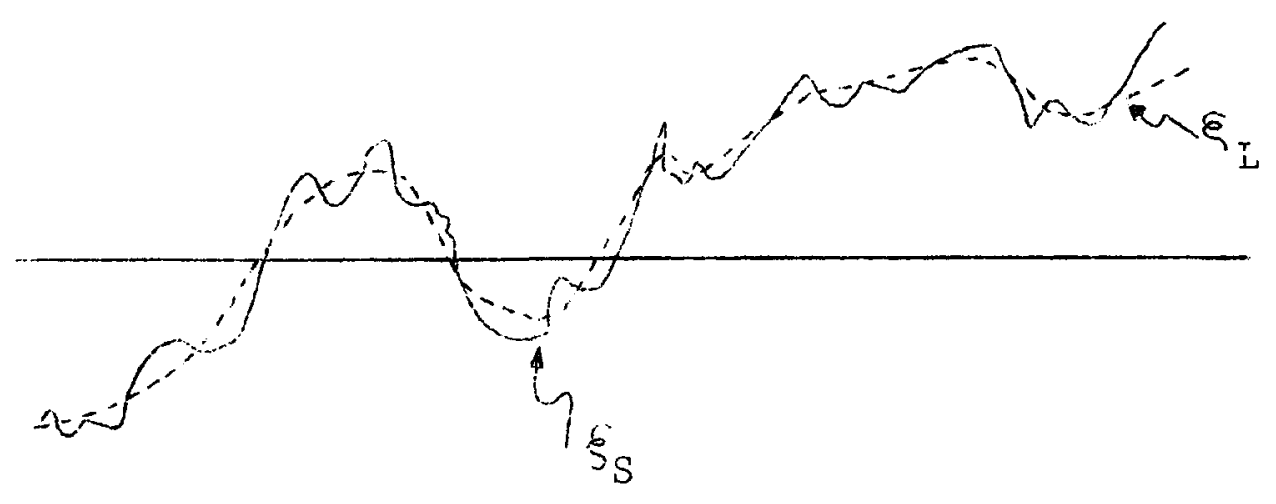

Fig. 2 


\title{
CRES LABORATORIES
}

\author{
Chemical Engineering Low Temperature Loboratory \\ Remote Sensing Laboratory \\ Electronics Research Laboratory \\ Chemical Engineering Heat Transfer Laboratory \\ Nuclear Engineering Laboratory
}

Environmental Health Engineering Laboratory

Digital Computer Technology Laboratory 
